\title{
HIERARCHICAL PRINCIPLE IN THE PROBLEM OF A TYPOLOGICAL SYSTEM DEVELOPMENT
}

\author{
Valentina Kamenskaya \\ Herzen State Pedagogical University, St. Petersburg, Russia \\ E-mail: kamenskaya-v@mail.ru \\ Larissa Khokhlova \\ Northern State Medical University, Arkhangelsk, Russia \\ E-mail: Ikhokhlova@rambler.ru
}

\begin{abstract}
The study contains the theoretical review of different historical approaches to the study of typological properties of a man. One of the well-known typological systems in foreign psychology has been developed in constitutionology, juvenility characteristics, however, haven't been included in its structure. On the basis of the studied samples the authors of the research consider the empirical proofs of juvenility existence, its significance in typological perspective and the formation of mental functions. The research findings of typological properties of the morphofunctional brain organization as a subject of both Russian and foreign psychology and psychophysiology are analyzed. Theoretical and experimental material is integrated in the analysis of individual typological peculiarities of students of higher educational establishments. The regularities of their manifestation at genotypic, behavioral and personal levels representing various hierarchical layers of a person's typological system are revealed.
\end{abstract}

Key words: behaviour, brain, constitution, hierarchy of topological properties, interhemispheric relations, juvenility, model, personal features.

\section{Introduction}

Being an important aspect of differential psychophysiology and psychology the investigations of systemic typology don't lose their relevance for several centuries. According to the system and classification view points the entire history of a man's study is the "arena" of numerous methodological approaches. In the chaos of individual variations of behavior and reflex reaction scientists try to reveal those determining factors of behavioral identity that are capable of composing the foundation of typological classifications.

The study of typological peculiarities began with Hippocrates-Galen's humoral theory based on four humors of the organism. In the Middle Ages differences between people were attributed to the prevalence of alchemical elements in the body. The epoch of Renaissance was marked by the appearance of the anatomical approach. Its supporters began studying the differences of tissues and width of the lumen of vessels. Followers of the endocrine theory appeared at the beginning of the $20^{\text {th }}$ century associated the peculiarities of the man's behavior with the balance and work coordination of the sympathetic and parasympathetic divisions of the vegetative nervous system. After Pavlov's research (1950) special emphasis began to be attached to the manifestation specificities of the properties of the nervous system that allowed to compare them with temperament types. From the structural and system approach suggested by Nebylitsin (1976) two major 
constituents of the multilevel organization of the nervous system properties began to be spoken about: the level of the nervous elements (neurons) and the level of the structural complexes of the brain. The investigations of the followers of the behavioral approach (Rusalov, 1991; Golubeva, 2005) were aimed at studying the dynamic side of the temperament, its intellectual, emotional and psychomotor activity. The idea of the man's complex study and his level organization with the basic genetically determined individual level belongs to Ananiev (2001) the founder of the psychophysiological school.

The presence of such a great number of approaches points to the complexity of personality typologies. At the same time the undying interest of scientists to this problem indicates its great scientific significance.

Permanent interest to the study of identity bases reflects not only the tendency to a more successful prediction of the peculiarities of individual behavior, but certain transformation in the understanding of the nature and essence of the individuality. The idea of the identity as a source of imperfections of scientific observations and evolution by-product is steadily being changed by the understanding of the identity significance as a necessary and natural result of the evolutionary development, foundation and driving force of various types of communication and uniting people (Medzhidova, 2007).

The main problem of the given research concerned the development of an integral model of a person's typological characteristics.

\section{Methodology of Research}

\section{General Background of Research}

The research was conducted at the Department of Psychology and Psychophysiology of a Child of Herzen State Pedagogical University of Russia (HSPU) and the Foreign Language Department of the Northern State Medical University (NSMU). The objective was twofold. One aim was to reveal the individual typological peculiarities in students of higher educational establishments. On the other hand it was necessary to see how these characteristics were manifested at genotypic, behavioral and personal levels and if there was any hierarchical interrelation between levels of a person's typological system.

\section{Sample of Research}

Studies were performed on groups of students studying psychology and students with different levels of proficiency in foreign language (well and badly advanced students). The sample size was 800 students of both sexes. The mean age ranged from 17 to 19 years. All participants gave informed consent. Study protocols were approved by Ethics Committees of HSPU and NSMU.

\section{Instrument and Procedures}

The research included both psychophysiological and psychological methods. The psychophysiological part of the study consisted of 1) EEG methods of brain investigation on a 16-channel "Neiron Spectr 3" electroencephalograph using the International 10-20 system of electrode placement and monopolar technique; 2) the authors' program of the reflexometric investigation (Kamenskaya \& Tomanov, 2007);3) the method of the experimental study and parameterization of the decision-making mechanism developed on the basis of Anokhin's theory (1980) of functional systems (investigation on a psychophysiological complex "Binatest"); 4) the anthropometrical express method of juvenility assessment including the measurement of the head circumference or the length of the cerebral skull on the sagittal line.

The psychological part of the study was aimed at the determination of personal characteristics (Kettel's test) and property assessment of the higher nervous activity (Strelau's test). 
The statistical analysis package Statistica 8 (for Windows, V 8.0, StatSoft) and MatLab (R2007b) were used for data analysis. For different experimental tasks and psychological tests sigma (statistical index of the variability), means and standard deviations were calculated. All measurements were estimated for compliance to the normal law of distribution. The obtained data were statistically analyzed by using Student t-test and calculating nonparametric correlations (Spearman correlation coefficient). For all analyses a P-value $\leq 0.05$ was considered statistically significant.

The EEG data recorded from each participant were visually inspected and trials were rejected when contaminated by artifacts induced by eye movements and muscle tension. Functional brain asymmetries were statistically analyzed according to coherence readings. Absolute spectral power was taken into consideration while determining hemisphere dominance.

Juvenility level was determined by the following formula: the mean + sigma $=$ an average juvenility level. Deviations from the mean (higher, lower) + sigma were considered as a high juvenility level or a low juvenility level correspondingly.

\section{Results of Research}

The main choice made in favor of students of two higher educational establishments (HSPU and NSMU) isn't casual. University training often proceeds in conditions of the increased environmental uncertainty and intensive cognitive stress. It's accompanied by a constant change of situational afferentation resulting in alteration of the dominant functional systems composing the student's academic activity. Its success is greatly determined by individual peculiarities including genotypic preconditions of abilities, mental state and the condition of physiological functions.

Structure determination of the typological properties can give some guidelines for psychological support of students during their university training. Following the idea that people with different typological features can be reliable in their activity and only certain ones make a person unreliable, we demonstrate the findings of the investigations conducted in groups of students with high and low levels of academic achievements. Typological properties are considered in the form of three hierarchically connected levels: genotypic (individual, constitutional), behavioral (temperamental) and phenotypically-personal.

The analysis of scientific literature, experimental works of Russian anthropology and psychophysiology allowed to develop the model of four-coordinate typological system of comparatively autonomous genotypically determined constitutional properties. According to theoretical conceptions (Rudkevich \& Kamenskaya, 1999; Ananiev, 2001; Rudkevich, 2001). this individual constitutional level includes somatotypological axis (mesomorphia, ectomorphia, endomorphia), the axis of biological maturation speed (retardation, acceleration), juvenility axis and the axis of the morphofunctional brain properties in the form of interhemispheric relations and general properties of the nervous system (strength, steadiness, speed, stochasticity)

Considered category of juvenility representing one of the man's constitutional properties, the principles of his biological organization and evolution playing a significant role not only in the organism formation but psyche as well emphasizes the appropriateness of the undertaken researches. The analysis of juvenility as a less studied constitutional property manifesting in interhemispheric relations and their manifestations in personal features and behavior is a key aspect.

The study of correlations of different juvenility levels with the quality of the sensorimotor integration assessed by the method of stochastic reflexometry (Kamenskaya \& Tomanov, 2007) has given some interesting findings.

Based on the juvenility level three groups were formed. They were: female students with a low juvenility level $(n=20)$, those with an average juvenility level $(n=77)$ and the ones with a high juvenility level $(\mathrm{n}=29)$.

The quality of the sensorimotor integration and speed characteristics of the sensorimotor reaction were assessed in two sensory tasks differing in their complexity degree. There was a signal detection in the speed task. It was necessary to carry out the motor action with the greatest possible speed to all incoming stimuli (visual stimuli of three colors with a total presentation probability 0.75 and 
acoustic stimuli with the appearance probability equal to 0.25 ). In differential more complex task it was necessary to execute a choice reaction to different visual stimuli: stimuli of red color required inhibition of the reciprocal motor reaction with the preservation of high-speed motor actions to other stimuli. Stochastic mode in these two tasks was fractal.

In addition to stochastic reflexometry we used a more complex perceptual task of the search of figure stimuli on a certain color background changing under the law unknown to the examinee. This dynamic matrix had four color backgrounds that changed and required voluntary attention distribution to the background color and to the search of the necessary figure in the matrix. Each correct answer changed the figure location in the matrix. Its volume was rather large ( 100 symbols -25 by each color). After each correct answer the number of symbols increased by one. Incorrect figure or incorrect color background were considered as mistakes. In case of any mistake there was a sound signal. The examinee had to remember the last figure and pay attention to the color background. After mistake correction the dynamic mode was restored and the work proceeded as before.

The verified hypothesis was that female students with different juvenility levels were characterized by the presence of differential features in the system of the sensorimotor integration, perceptual and motor activity. Girls with a higher juvenility level are likely to have more effective mechanisms of the sensorimotor reaction and high level of the selective attention organization as compared with girls with a low juvenility level. At the same time, the developed juvenility feature must also determine the advantage at a perceptual-cognitive level manifesting in faster and better performance (with a less number of mistakes) of perceptual tasks on figure recognition in the dynamic matrix.

It should be noted that girls with low and high juvenility levels carry out simple tasks on stimulus detection almost equally. They have close average values of the response time (RT) to all sensory stimuli. The RT to sound tends to be longer in girls with a high juvenility level. The task complication at the expense of the choice reaction emphasizes the ratio of the response quality in girls with different juvenility levels as shown in Figure 1.

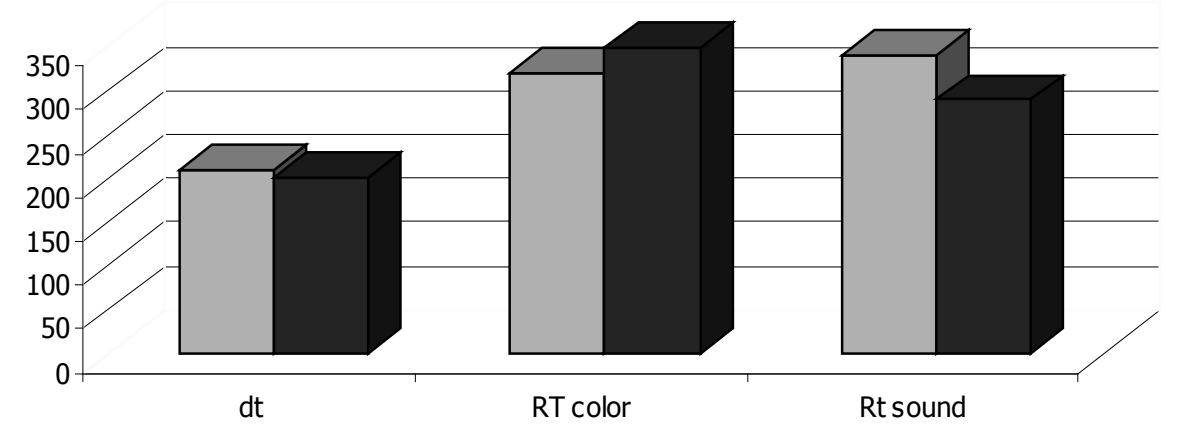

Figure 1: Speed parameters of the performance of the complex choice task.

Note: $d t$ - mean value of the RT to all sensory stimuli both visual and acoustic. RT color - mean value of the RT to all visual stimuli. $R T$ sound - mean value of the RT to an acoustic stimulus. Left histograms in pairs-parameters of girls with a high juvenility level, right histograms in pairs - parameters of examinees with a low juvenility level.

Girls with different juvenility levels have similar reactions to sensory stimuli, the RT to the acoustic stimulus being significantly longer in girls with a high juvenility level as compared with the RT characteristic of the girls with a low juvenility level. Students with a high juvenility level have quicker reactions to highly probable stimuli (stimuli with a high appearance frequency), the visual ones in the given task. They either miss signals with a low appearance frequency or have delayed reactions to them. It means that at the level of the signal detection and choice reaction execution the girls with a high juvenility level seem to be worse in the reaction speed than their peers with a low juvenility level. They are therefore characterized by less perfect mechanisms of the selective attention in comparison with the girls with a low juvenility level.

Different situation is observed in case of the performance of perceptual tasks of figure recognition on the background of different color. Girls performed two identical tasks of the figure search on a changing color background with a break to test their motivational characteristics. During this 
interval the girls were asked to assess the time of the task performance. Several simple and clear facts were received. Irrespective of the juvenility level girls learned in the course of the task performance. During the second test they all had less time expenditure to the task performance with a less number of mistakes as shown in Figure 2.

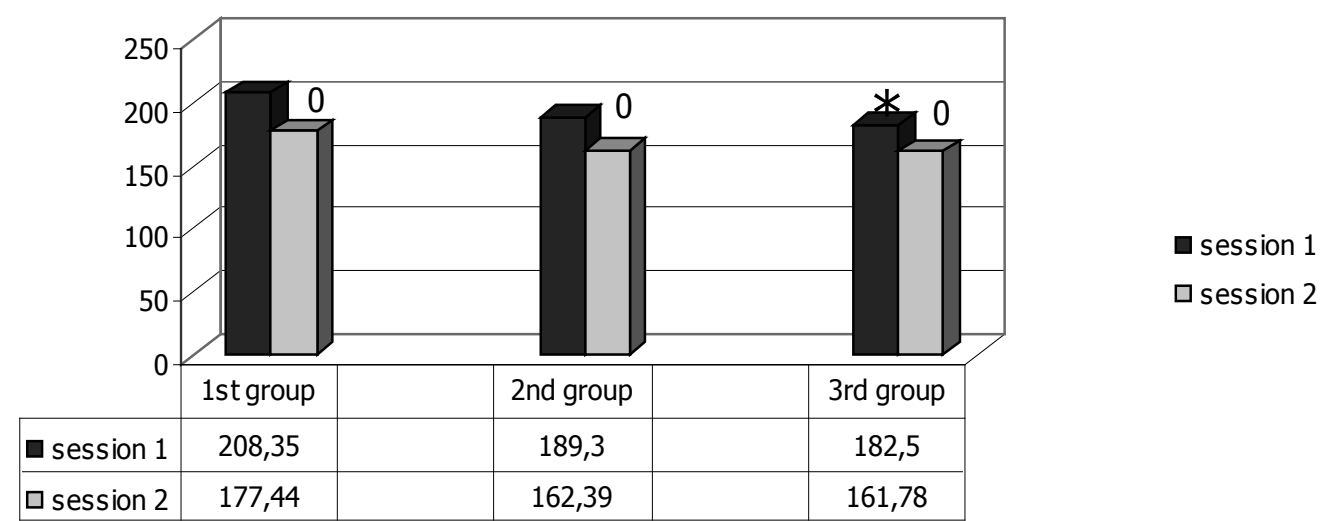

\section{Figure 2: Mean time spent for test implementation.}

Note: " 0 " - reflects a significant decrease of time expenditure characteristic of all girls in the second session as compared with the first one. "*" - reflects significant differences of time expenditure in girls with a high juvenility level ( $3^{\text {rd }}$ group) in comparison with those with a low juvenility level ( $1^{\text {st }}$ group).

Figure 2 indicates higher speed of processes of the figure recognition and less time expenditure to the matrix performance both in the first and second sessions in students with a high juvenility level in comparison with those with a low juvenility level. Higher speed of the cognitive and search activity based on the random access memory, voluntary attention concentration and its switching corresponds to a less number of mistakes made by the female students with a high juvenility level in comparison with their peers with a low juvenility level. Thus, in more complex perceptual and cognitive task the girls with a high juvenility level appeared to be more successful as compared with the girls with a low juvenility level. Research findings (Kamenskaya \& Pavlov, 2010) have concluded that it can be considered as a manifestation of more perfect mechanisms of the random access memory and voluntary attention.

The carried out statistical analysis indicates some degree of autonomous functioning of the genotypic level (EEG results, assessment results of the nervous system properties) composing $57.4 \%$ of general dispersion. The levels of temperamental (investigation on a psychophysiological complex "Binatest", results of Strelau's test) and personal properties (results of Kettel's test) make up 45.7\% and $37.4 \%$ correspondingly.

External factors are well known to influence the psyche formation. When considering this thesis from the psychophysiological investigations view points and taking into consideration the obtained interlevel gap we must ask the following question - what external factors and through what internal conditions influence the realization of the genotypic development program and manifestations of genotypically determined psychological and psychophysiological characteristics of the person's individuality.

It is hypothetically possible to believe that such conditions are the brain systems providing mental functioning. Khomskaya (2000) gives a number of reasons to suggest that neuropsychological factors reflect the genetic mechanisms of mental activity, i.e. genotype influences the mental phenomena through morphofunctional brain formations.

Conducted EEG studies (Khokhlova \& Deryagina, 2005; Khokhlova, 2011) have systematized data related to the structurally functional features of the cortical activity of students with high and low levels of academic achievements. Parameters of the alpha rhythm seem to be the most interesting ones in our case. Reviews of research (Annet, 1995; Andersen, 2003; Dumenko, 2007) show that alpha band is genotypically determined The studied groups differed in the power of biopotentials of the background alpha band in the frontal and parieto-occipital areas of the left hemisphere (well advanced students) and in the fronto-occipital leads of the right hemisphere (badly advanced students) (Figure 3). 
Well advanced students

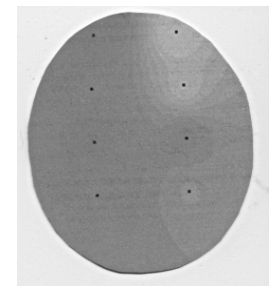

Badly advanced students

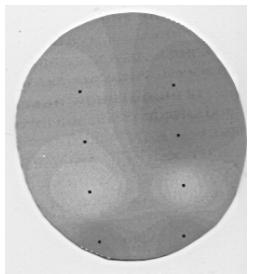

\section{Figure 3: Map of the spectral power of the background alpha band in groups of well and badly advanced students.}

Note: darker red color - the greatest spectral power

The greatest concentration of the cross-correlation associations of the cortical potentials at rest is registered in these areas. Sviderskaya (1990) points to the existence of the "cognitive axis" including the abovementioned brain areas taking part in the analysis and synthesis of information in different ways (successive and simultaneous) and at various levels of consciousness. It was shown (Sviderskaya \& Korolkova, 1994) that the "cognitive axis" was a genetically determined manifestation of the space organization of the cortex biopotentials. The location of the hyperactivity areas can vary depending on the physical condition of a man. Violation of the cortical, subcortical interrelations is considered as inversion of the "cognitive axis" representing the defect in the system of extero- and interoceptive information processing (Kiroi, 1991).

At a neurophysiological level the activity of the frontal and parieto-temporal areas of the right hemisphere is associated with the system of the avoiding behavioral inhibition and high levels of personal anxiety (Aftanas, 2005, p. 325). The prevalent activity of the parieto-occipital area of the left hemisphere corresponds to a positive emotional condition that is likely to demonstrate the influence of the genotypic factor on the phenotypic manifestation of anxiety. The investigation into EEG organization (Shepovalnikov \& Tsitseroshin, 2007) indicates the presence of some association between the nature of the regional cortical activity, functional brain asymmetry and personal specificities of emotional functioning

According to research findings direct relation between the parameters of situational anxiety, personal anxiety and alpha-band amplitude in the frontal areas of the right hemisphere was revealed in badly advanced students. These data are likely to indicate some disadaptation manifestations in this group of students.

Proved interrelation of cognitive and emotional processes with the alpha-band activity emphasizes the role of their integration at the cortical level.

Optimal EEG parameters of the alpha band observed in the group of well advanced students correlated with significantly $(\mathrm{p} \leq 0.05)$ greater indices of the mobility and steadiness of the nervous processes (Strelau's test) reaching $66.5 \pm 0.57$ points on a mobility scale and $0.94 \pm 0.01$ points on the ratio of excitation and inhibition processes.

In the group of badly advanced students the results of Strelau's test were the following: mobility $-54.2 \pm 1.33$ points; steadiness $-0.80 \pm 0.02$ points. The steadiness of the nervous processes in this group of students demonstrated the negative interrelation with the activity of the sensory areas of the cortex ( $\mathrm{r}=-0.97)$ indicating the low threshold of sensory excitation. The results obtained let us speak about the individual modulation of the intensity of the anterior and posterior focuses of the bioelectrical activity of the brain depending on the type of the higher nervous activity.

Concerning the behavioral (temperamental) level considered as the following step of the identity typology, the method results of the experimental study and parametrization of such fundamental mechanism of the higher nervous activity as decision-making in the conditions of a choice from some alternative purposeful reactions (psychophysiological complex "Binatest", Russia) seem to be the most interesting.

The statistical analysis of our research findings showed the heterogeneity of the obtained data that in turn dictated the need of the identification of certain typological features.

While investigating on the psychophysiological complex "Binatest" the mode without behavior 
determination by external stimuli when the examinee makes a multiple "free choice" should be mentioned. The decision-making process determined by the internal mechanisms of the successive aim Volume 5, 2013 formation only is manifested in this case. As a made choice is usually dichotomic we used the situation of the choice from two alternative reactions - pressing by a computer mouse the right or the left button. By means of the computer mouse the examinee had to light two boxes in the sequence and with a speed that seemed to be the most convenient for him or her.

Two opposite groups were revealed. They are students preferring "a single choice" (one button pressing) and students preferring "a repeated and $\mathrm{N}$ times in succession choice". The carried-out statistical analysis has revealed two groups of orthogonal factors characteristic of the general structure of test parameters.

The first group was made up by the structural indices (choice preference) determining $43.57 \%$ of the general dispersion. The second group included dynamic (high-speed) parameters (15.73\%). Thus, the choice preference appeared to be dominant in the general architecture of the behavioral act in the conditions of free activity. Structural variations determined the difference in dynamic characteristics of the studied groups. Maximal lability (or minimal mean time between reactions) was noted in students preferring "a repeated and $\mathrm{N}$ times in succession choice" $(0.30 \pm 0.01 \mathrm{~s}$. against $0.57 \pm 0.03 \mathrm{~s}$. in the group of students preferring "a single choice"- one button pressing) that was likely to indicate the high speed of the nervous processes of the frontostriated system realizing the purposeful behavior (Simonov, 1997).

According to the differential indices of the decision-making time at a repeated choice we assessed such functional patterns of the behavioral act as a mean time of the choice repetition reflecting the information component and the mean time of the choice change - the dynamic constituent of the motivational component, the search activity in particular.

In accordance with Simonov's theoretical conception (1997) the presence of a certain dominant need (motivational component) and the prediction of the probability of this need achievement (information component) are the behavior determinants. The prediction success is determined by the man's knowledge of the environment. The motivational components are mainly manifested in the degree of the search activity and depend on the signals of the environment. It should be noted that it is the search activity that represents a general nonspecific factor determining the body's resistance to stress and harmful effects at various forms of behavior (Rotenberg \& Arshavsky, 1994).

According to our research findings both the information (mean time of the choice repetition) $(0.50 \pm 0.04 \mathrm{~s}$.) and motivational (mean time of the choice change) $(0.48 \pm 0.03 \mathrm{~s}$.) components are equally demonstrated in the structure of the behavioral reactions of students preferring "a repeated and $\mathrm{N}$ times in succession choice". The information component indices composing the successful activity basis were slightly higher although these differences were not statistically significant. The excess manifestation of the search activity (dynamic constituent of the motivational component $0.66 \pm 0.05$ ) indicating the high level of the behavior uncertainty was noted in the group of students preferring "a single choice".

Considering the above, it should be noted that behavior strategies depend on the man's individual specificities. Behavioral (temperamental) level representing certain sequence of reactions developing in time and space determines steady forms of the man's behavior in standard conditions. The properties of the nervous system manifesting in the functional brain activity, its greater or less excitability, the mobility of the nervous processes and speed of the formation of temporal associations mediate the brain neuronal activity and psychological characteristics demonstrating the possible influence of the genotypic specificities on the human behavior.

The third layer in the typological hierarchy is phenotypically personal. According to research findings (Khokhlova, 2011) it is manifested in specific characteristics of a person's activity and is influenced by the brain functional peculiarities.

Important information was obtained while analyzing the correlation structure of the personal characteristics determined by Kettel's test and alpha-band amplitude in standard leads.

The left-hemisphere dominant students ( $85 \%$ of well advanced students and $37 \%$ of badly advanced students) had a number of distinctive features. Such personality properties as increased self-control level (factor Q3), courage (factor H) and self-sufficiency (factor Q2) had positive correlations with the activity of the sensory areas of both hemispheres (Table 1). Parameters of the Q4 factor (tension) correlated negatively with the activity of the sensory and association areas of the right hemisphere. 
Factor $\mathrm{C}$ (emotional stability) had positive correlations with the activity of the frontal integrative areas of the cortex. The prevalence of positive correlations is likely to indicate a sufficient level of the cortical control in left-hemisphere dominant students. Evenly distributed loading between brain hemispheres prevents the appearance of the failure of the central mechanisms of regulation. In this case it's possible to say about the effective adaptation strategies characteristic of such students.

Table 1. Correlation structure of Kettel's test parameters with alpha-band amplitude in standard leads.

\begin{tabular}{lclc}
\hline \multicolumn{2}{c}{ Left-hemisphere dominance } & \multicolumn{2}{c}{ Right-hemisphere dominance } \\
\hline Parameter & $\mathrm{r}$ & Parameter & $\mathrm{r}$ \\
\hline Factor Q3 - F3 & 0.67 & Factor Q3 - F3 & -0.95 \\
Factor H - F4 & 0.72 & Factor Q3 - F7 & -0.97 \\
Factor Q2 - 01 & 0.77 & Factor Q3 - C4 & 0.93 \\
Factor Q4 - T6 & -0.68 & Factor H - F3 & -0.86 \\
Factor Q4 - P4 & -0.66 & Factor Q4 - F8 & 0.71 \\
Factor C - FP1 & 0.74 & Factor Q1 - F4 & 0.69 \\
Factor C - F7 & 0.82 & & \\
Factor C-P3 & 0.87 & & \\
\hline
\end{tabular}

The correlation structure observed in the right-hemisphere dominant students $(15 \%$ of well advanced students and $63 \%$ of badly advanced students) was characterized by the prevalence of negative correlations with the scales reflecting both sthenic (impulsation - increased self-control - factor Q3; shyness - factor $\mathrm{H}$ ) and hyposthenic response type (tension - factor Q4; introversionextroversion - factor Q1). It is likely to point to the fact that behavior strategies of the righthemisphere dominant students are determined by a combination of two variables: the tendency to self-actualization and tendency to the increased self-control to keep relations with people around. The noted low background activity of the alpha-band in this case seems to be a compensatory mechanism that prevents the transformation of the emotional intensity into a biological variant of disadaptation (Sobchik, 1998).

The findings obtained emphasize the complexity of the cause-effect relationships between the genotype and phenotype with respect to the characteristics reflecting the social personality status, i.e. her or his social genesis. All these personal specificities have natural basis due to the typological manifestation peculiarities of the properties of the nervous system. On the other hand they are the result of the development "trajectory" following the interaction regularities of the genotype and the environment.

\section{Discussion}

Typological properties of a person have a complex hierarchical structure with closely interrelated levels. It's a specific map of the psyche and human nature. Available typological systems are based on research findings in the field of somatotypology as a part of constitutionology (Sheldon, 1940; Conrad, 1963; Krechmer, 1995), biochemistry (Buss, 1984), psychophysiology (Riley, 2002), genetics (Rushton, 1986; Plomin, 1989).

Our approach to the typological system is the one of modern psychology and psychophysiology. The suggested three-coordinate system with genotypic, behavioral and personal levels seems to be more appropriate to understand preconditions of cognitive abilities and human behavior specificities. Such constitutional characteristic of a person as juvenility proved to have great significance in the properties of the nervous system. Its effect is obvious in mental processes, quantitative and qualitative parameters of the sensorimotor response.

Research findings indicate that adult girls with a high juvenility level lose their advantage in 
the speed and quality of the sensorimotor integration. They focus on highly probable signals and miss the ones with a low appearance frequency. It's likely to indicate the decrease of the selective attention switching. At the same time adult girls with a high juvenility level are characterized by high speed and good accomplishment quality of the perceptual and cognitive tasks, i.e. tasks of higher complexity degree in comparison with the tasks of stimulus detection and choice reaction execution. Thus, at the level of highly organized functions the individuals with a high juvenility level have advantage over those with a low juvenility level.

The results obtained are consistent with the experimental material related to the correlations between different levels of juvenility and teenagers' psychological features (Rudkevich, 2001). It was shown that teenagers with a high juvenility level had high or average-to-high intelligence, decreased aggressiveness and vague leadership. In the majority of cases they have introvertive character. Teenagers with a low juvenility level are characterized by opposite features. They are rather aggressive and predisposed to physical violence; the level of intelligence development is lower as compared with their peers with a high juvenility level.

Typological characteristics are manifested in the interhemispheric relations, personal features and decision-making mechanisms. The formation of the behavior vector is determined by the functional (dominant) condition of the central mechanisms of the brain. Ukhtomsky (1966) reached the conclusion that the dominant was based on three major factors: prevalent motivation, last life experience and the assessment of the stochastic structure of the environment. According to the research (Batuev, 1981) the first two factors are the internal behavior determinants creating the mosaic of the functional conditions of the brain structures

Based on the above it seems possible to consider the genotypic level as a key aspect in a person's development. The investigation of the genotypic level determinants becomes a special link binding the individual genome and individual specificities of the man's psyche. It explains the strategy of our researches, namely: the approach to the study of the determination of typological peculiarities through the assessment of the genotype role in the variability of psychophysiological and psychological features.

\section{Conclusions}

On the basis of research findings it's possible to conclude that among otherwise healthy people there is an internal biological heterogeneity determining the choice of behavior strategies. In our investigations we've tried to develop a sufficiently valid individual typological approach with respect to the prognosis of the person's adaptive ability and his or her success.

The training process at a higher educational establishment is characterized by stochasticity and unpredictability of the interaction of the regulatory functional systems. The properties of the genotypic, temperamental and personal levels represent the invariant slowly changing parts of the system of typological peculiarities of a person or the ones that remain constant for a long time. Their complex analysis becomes a necessary condition of the prediction of dynamics and efficiency of the behavioral reaction in changing environmental conditions as well as psychological support of students during their university training.

The results achieved give us very positive feedback to proceed with the research of personality typology.

\section{References}

Ananiev, B. G. (2001). A man as a subject of cognition. St. Petersburg: Peter

Aftanas, L. I. (2005) Peculiarities of the interhemispheric distribution of the EEG spectral power in highly anxious individuals in emotionally neutral conditions and at negative activation. Journal of the Higher Nervous Activity, 3,322-328.

Andersen, P. (2003). Physiological basis of the alfa rhythm. N.Y.: IOSMI.

Annett, M. (1995). The Right-shift theory of a genetic balanced polymorphism for cerebral dominance and cognitive processing. Current Psychology of Cognition, 14 (5), 427-480. 
OF PSYCHOLOGY

IN THE $21^{\text {st }}$ CENTURY Volume 5, 2013

44

Buss, D. M. (1984). Evolutionary biology and personality psychology: Toward a conception of human nature and individual differences. American Psychologist, 39, 361-377.

Conrad, K. (1963). Der Konstitutiontypus. Berlin-Gottingen-Heidelberg.

Dumenko, V. N. (2007). Training and high frequency constituents of the electrical brain activity. Russian Academy of Sciences, the Institute of Higher Nervous Activity.

Golubeva, E. A. (2005). Abilities, personality, individuality. Ed.: FENIKS.

Kamenskaya, V. G., \& Pavlov, K. I. (2010). Psychophysiological characteristics of cognitive functions in 18-23 year old girls with different juvenility levels. Psychology of Education in Poly-cultural Field, 3 (3), 24-34.

Kamenskaya, V. G., \& Tomanov, L.V. (2007). Psychophysiology and intellectual activity. Theoretical and experimental researches. Elets: EGY.

Khokhlova, L. A., \& Deryagina, L. E. (2005). The influence of the higher mental functions of a man on the foreign language mastering. Proceedings of the All-Russian Conference "Problems of Education". Arkhangelsk.

Khokhlova, L. A. (2011). Manifestation of the functional brain asymmetry peculiarities in the mechanisms of the mental processes organization. Bulletin of Orlov State University, 1, 305-307.

Khomskaya, E. D. (2000). Neuropsychology and psychophysiology of individual differences. Orenburg: OOIPKRO

Medzhidova, S. M. (2007). Up-to-date approaches to the psychological personality typology. Psychological Center [CD-ROM]

Nebylitsin, V. D. (1976). Psychophysiological investigations of individual differences. Moscow: Publishing House.

Pavlov, I. P. (1950). General types of the higher nervous activity of animals and a man. Physiology of the Higher Nervous Activity, 2, 17-35.

Riley, M. A. (2002). Variability and determinism in motor behavior. Journal of Motor Behavior, 2, 99125.

Rotenberg, V. S., \& Arshavsky, V. V. (1994). Search activity and adaptation. Moscow: Science LTD.

Plomin, R. (1989). Environment and genes: Determinants of behaviour. American Psychologist, 44, $105-$ 111.

Rudkevich, L. A., \& Kamenskaya, V. G. (1999). Four-coordinate model of the constitutional organization of the individual. Proceedings of the International Scientific Conference: Sciences and Humanities. St. Petersburg.

Rudkevich, L. A. (2001). Theoretical experimental bases of the age and differential psychosomatology. (Doctoral dissertation, Herzen University, St. Petersburg).

Rusalov, V. M. (1991). Biological principles of individual psychological differences. Psychological Journal, $12(5), 3-17$.

Rushton, J. P. (1986). The heritability of individual differences. Journal of Personality and Social Psychology, 50, 1192-1198.

Sheldon, W. H. (1940). The varieties of human physique: An introduction to constitutional psychology. New York: Harper \& Brothers

Shepovalnikov, A. N., \& Tsitseroshin, M. N. (2007). Analysis of the spatio-temporal EEG organization the way to knowledge of neurophysiological mechanisms of the integrative brain activity. Journal of the Higher Nervous Activity, 57 (6), 663-670.

Sobchik, L. N. (1998). Introduction to personality psychology. Theory and practice of psychodiagnostics. Moscow: Institute of Applied Psychology.

Sviderskaya, N. E. (1990). Coherent structures of the electrical activity of the brain cortex. Human Physiology, $16(3), 12-18$.

Sviderskaya, N. E., \& Korolkova, T. A. (1994). Space organization of the electrical brain processes. Electroencephalography and Clinical Neurophysiology, 4 (11), 110-123. 
Simonov, P. N. (1997). Brain mechanisms of emotions. Journal of the Higher Nervous Activity, 47 (2), 320-328.

Батуев, А. С. (1981). Высшие интегративные системы мозга. Ленинград: Наука.

Кирой, В. Н. (1991). Механизмы формирования функционального состояния мозга человека. Ростов н/Д: Изд-во РГУ.

Кречмер, Э. (1995). Строение тела и характер. Москва: Педагогика Пресс.

Ухтомский, А. А. (1966). Доминанта. Москва: Наука.

Advised by Jüratè Armoniene, Vilnius University, Lithuania

Received: January 30, 2013

Accepted: March 14, 2013

Valentina Kamenskaya Ph.D. (senior doctorate) in Psychophysiology, Professor, Corresponding Member of the Russian Academy of Education, Herzen State Pedagogical University, St. Petersburg, Russia.

E-mail: kamenskaya-v@mail.ru 